\title{
Posibilidades del mecanizado por ultrasonidos rotatorio para fomentar el uso de las cerámicas técnicas en el mercado Español actual
}

\author{
I. ARRIOLA ${ }^{1}$ O. GONZALO ${ }^{1}$ Y I. BENGOETXEA ${ }^{1}$
}

`Unidad de Tecnologías avanzadas de fabricación, Fundación Tekniker-IK4, CIC marGUNE, Avenida Otaola 20, 20600 Eibar, Guipúzcoa

\begin{abstract}
Las propiedades mecánicas de las cerámicas técnicas son adecuadas para aplicaciones en diferentes sectores como la medicina (prótesis,...), automóvil (rodamientos,....), aeroespacial (loseta,...), óptica (láser,...), etc. donde se requieren características como la gran resistencia al desgaste, alta dureza y estabilidad termoquímica. Sin embargo, la fabricación de estos materiales es difícil y costosa, en algunos casos alcanzando el 90\% del coste final, por lo que su aplicación está limitada.

Este trabajo presenta el mecanizado por ultrasonidos rotatorio (RUM) como un proceso no convencional que permite la fabricación de piezas cerámicas con geometrías 3D complejas de manera ecológica y económica. Este método consiste en la eliminación de material utilizando una herramienta superabrasiva dotada de 3 movimientos: rotación, vibración axial ultrasónica $(20 \mathrm{KHz})$ y avance contra la pieza fija. Este proceso combina el rectificado convencional y el mecanizado por ultrasonidos logrando mayores volúmenes de material evacuado manteniendo las fuerzas de corte bajas. De esta manera, se superan las limitaciones de los métodos convencionales utilizados actualmente, obteniendo procesos más precisos, flexibles y económicos.

En definitiva, el RUM es un proceso prometedor para las operaciones de mecanizado de cerámicas estructurales. Sus características permitirán fomentar el uso de las cerámicas en un mayor número de aplicaciones industriales.
\end{abstract}

\section{Palabras clave: Aplicaciones estructurales, mecanizado, ultrasonidos, cerámica}

Possibilities of rotary ultrasonic machining in order to give a boost to technical ceramics in the actual Spanish market

The advanced properties of engineering ceramics are suitable to meet the requirements of several engineering applications such as medical (prosthesis...), automotive (bearing...), aerospace (tiles,...), optics (lasers,...) and so on, where high hardness, high wear resistance and thermo chemical stability are needed. However, the processing of these materials is difficult and costly, in some cases up to $90 \%$ of the price, which leads ceramics to limited applications.

Rotary Ultrasonic Machining (RUM) is presented as a non conventional environmental-friendly and cost effective process that enables to obtain complex 3D shape ceramic products. A rotating tool with metal-bonded diamond abrasives is ultrasonically vibrated (typically $20 \mathrm{KHz}$ ) and fed toward to the fixed workpiece at a constant pressure or a constant feed rate This process combines conventional grinding and ultrasonic machining, offering the possibility of increased material removal rates while keeping the machining forces low. Thus, the limitations of actually used conventional processes are overcome introducing more accurate, flexible and cost effective process capabilities.

On balance, RUM is a promising process for primary machining operations on structural ceramic components. Its features will lead to enhance the use of ceramics in more industrial applications by increasing product development/design capabilities, high added value products and sustainable process.

Keywords: Structural applications, machining, ultrasonic, ceramic

\section{INTRODUCCIÓN}

El incremento en la demanda de materiales con mejores propiedades mecánicas desde diferentes sectores como la medicina (prótesis,...), automóvil (rodamientos,....), aeroespacial (loseta,...), óptica (láser,...), etc. ha obligado al desarrollo de nuevos materiales que cumplan con esos requisitos mediante la investigación en la Ciencia de Materiales (1). Como resultado, por ejemplo, en el caso particular de las cerámicas se han obtenido materiales con características elevadas de gran interés para la industria $(2,3)$ :

- Resistencia a la fluencia térmica

- Resistencia al choque térmico

- Resistencia al desgaste, a la corrosión y a la cizalladura.
- Gran estabilidad química

- Alta dureza

- Amplia gama de cualidades eléctricas

- Elevada resistencia a compresión

Pero por otra parte, debido a estas buenas propiedades y comportamiento en las aplicaciones más exigentes, su transformación y procesado presentan problemas que limitan la precisión y elevan los costes de producción. Por ejemplo, en algunos casos alcanzando el $90 \%$ del coste final (4), por lo que su aplicación está limitada. Esto queda reflejado en los datos proporcionados por la Industria Cerámica Europea (Cerame-Unie) donde posiciona a las cerámicas técnicas en 
cuarta posición con un $9.5 \%$ de todas las ventas en la industria de la cerámica (5). Como resultado de los problemas citados, las cerámicas no han podido competir con otros materiales más comúnmente empleados (titanio, aceros endurecidos,...) impidiendo mostrar su potencial en el mercado actual.

El mecanizado de las cerámicas técnicas se realiza habitualmente empleando procesos de rectificado o electroerosión, y en promedio suponen entre el 30-60\% del coste total de producción. A pesar de la importancia de estos procesos, debido a sus capacidades, existen limitaciones técnicas a la hora de conseguir geometrías complicadas, tolerancias ajustadas y costes reducidos. Como consecuencia, el mecanizado de materiales duros y frágiles se ha convertido en un problema tecnológico y económico, que aún hoy en día se presenta como un reto importante.

Actualmente es de suma importancia aumentar el rendimiento en los procesos de fabricación para mantener la competitividad de las empresas y su posición de mercado. La necesidad de esta mejora es consecuencia de dos factores, por un lado, el económico asociado a la reducción de costes por la entrada de competidores ubicados en países donde los costes salariales son más bajos (6), y por otro lado, el tecnológico con los retos para la fabricación de productos con mayor valor añadido que incrementan la complejidad de los procesos de mecanizado (nuevos materiales, geometrías complejas, requisitos de calidad y precisión...). En definitiva, la supervivencia se reduce a la mejora y optimización de los procesos para fabricar más rápido, mejor y más barato para cumplir con las exigencias de costes, plazo y calidad que exigen los clientes.

Una alternativa es la utilización de procesos no convencionales como el mecanizado por ultrasonidos rotatorio (RUM); que superan las limitaciones de los métodos convencionales utilizados actualmente, permitiendo operaciones (taladrado, fresado, roscado, contorneado, etc) con mayor versatilidad y más libertad en el diseño de los componentes cerámicos. Esto posibilitará que las cerámicas técnicas amplíen su campo de actuación en más sectores mejorando las prestaciones actuales $(7,8,9,10)$.

Realizando una revisión bibliográfica, hasta hace unos años, los métodos más empleados para el mecanizado de las cerámicas técnicas eran el USM (Ultrasonic Machining o Mecanizado por Ultrasonidos tradicional) y el proceso de rectificado. Pero gracias a la introducción de la vibración axial en combinación con el proceso de rectificado con diamante se obtienen mejores rendimientos que en los citados procesos. A este nuevo proceso se le llamó mecanizado por ultrasonidos rotatorio, (Rotary Ultrasonic Machining, RUM). El RUM fue inventado en 1964 por Percy Legge, un técnico del "United Atomic Energy Authoriry" (11, 12, 13). Los primeros resultados experimentales relevantes se mostraron en un artículo de revisión del estado del arte por Z.J. Pei et al. (14). Desde entonces, se han analizado distintos materiales catalogados como difíciles de mecanizar: cerámicas técnicas (15), vidrios técnicos (16), composites(17), titanio (18), acero inoxidable (19), silicio (20), Germanio (21), $\mathrm{LiNBO}_{3}$ (22), Circonia (23), Cuarzo (24), etc (25).

En los cuales, los aspectos o parámetros más estudiados para determinar el rendimiento del RUM han sido el desgaste de la herramienta material, la tasa de arranque (Material Removal Rate, MRR) y rugosidad superficial (26, 27, 28, 29, 30, $31)$. Otros aspectos que hasta ahora no se han estudiado tanto pero están cogiendo más relevancia son la capa de tensiones residuales de compresión $(17,32)$ que podrían aumentar la vida a fatiga del componente mecanizado y las grietas en las piezas. Por ejemplo, Li et al. (33) diseñaron un modelo de elementos finitos 3D para predecir cuándo se van a dar las micro-roturas y lo verificaron experimentalmente.

Además de los análisis experimentales se han realizado trabajos de modelización del proceso RUM. Por ejemplo, Prabhakar et al. (34) desarrollaron un modelo teórico para calcular el MRR en el proceso RUM, fue el primer intento en caracterizar este parámetro. Desde entonces, los modelos se pueden agrupan en dos grupos: uno que se basa en un material frágil y el otro en un material dúctil $(35,36)$. Entre ellos, hay modelos teóricos que tienen en cuenta el principio de rotura por indentación (34) mientras otros se basan en modelos mecanísticos (37). Adicionalmente, se pueden encontrar trabajos relacionados con desarrollos para recubrir las herramientas de diamante (17).

Todos estos avances y estudios han hecho que, comparado con el mecanizado por ultrasonidos tradicional (USM), los beneficios que se pueden apreciar en el RUM se resuman en los siguientes aspectos $(29,38,39)$ :

- Rapidez 10 veces mayor.

- Mayor facilidad para taladrar pequeños agujeros, con notable mejora en la precisión.

- Menor presión en la herramienta y menor tasa de desgaste.

- Eliminación del fluido abrasivo.

Por otra parte, comparado con el rectificado, las ventajas del RUM son $(7,40)$ :

- Reducción de las fuerzas de corte (65\%-70\%) y del desgaste de herramienta.

- Mejora de la rugosidad superficial (29\%) (41).

- Eliminación de la zona afectada térmicamente, y de las alteraciones químicas en la superficie de la pieza.

En este trabajo se presenta el mecanizado por ultrasonidos rotatorio (RUM) como un proceso no convencional que permite la fabricación de piezas cerámicas con geometrías 3D complejas de manera eficiente y económica. El objetivo principal es el de analizar el rendimiento del proceso RUM a la hora de mecanizar Óxido de Aluminio (Alúmina) comparado con el rectificado. Primero se ha realizado un análisis individual de dos operaciones: taladrado y fresado, en condiciones de acabado, con objeto de analizar el efecto de las condiciones de corte en parámetros tales como las fuerzas de corte, rugosidad, redondez y desgaste mediante el método de diseño de experimentos (DOE) y el análisis de la varianza (ANOVA). Después, se ha mecanizado una pieza real para verificar que los resultados obtenidos en el análisis individual de cada proceso se reflejan en el componente que forma parte de un anillo de ajuste.

\section{MÉTODO EXPERIMENTAL}

\subsection{Descripción del proceso}

El RUM es un proceso que consiste en la eliminación de material utilizando una herramienta de material superabrasivo mediante la combinación de tres movimientos: 
rotación, vibración axial ultrasónica (frecuencia $20 \mathrm{kHz}$ y amplitud de 5-50 $\mu \mathrm{m}$ ) y avance contra la pieza. Este proceso utiliza herramientas abrasivas generalmente de diamante o CBN (electrodepositado o aglomerado). Por otro lado, el proceso utiliza taladrina bombeada a través de la herramienta para eliminar las virutas de la zona de corte, evitando el embotamiento y refrigerando el proceso (Figura 1, a).

El elemento principal de las máquinas de RUM es el cabezal ultrasónico. Este componente incluye una serie de cerámicas piezoeléctricas que convierten una señal eléctrica proveniente del generador de ultrasonidos en una vibración mecánica a la misma frecuencia (ver figura $1, b$ ).

Las cerámicas piezoeléctricas forman parte del denominado transductor, pero existen otros 2 elementos que conforman el conjunto resonante del cabezal. En primer lugar, el denominado booster, que es el responsable de la transmisión de la vibración desde el transductor hacia el sonotrodo o herramienta, pudiendo introducir una amplificación mecánica de la vibración ultrasónica. En segundo lugar, el citado sonotrodo o herramienta que es el elemento final del conjunto resonante y lleva la amplitud de vibración a la zona de trabajo produciendo la amplificación final (7). En la figura 2 aparece a continuación se muestra esquemáticamente el sistema resonante del cabezal ultrasónico:

\subsection{Metodología de trabajo}

La máquina de RUM empleada en este estudio es de Sauer Ultrasonic (DMG) modelo DMS 35 (Figura 3, a). Este incluye un generador de ultrasonidos y un cabezal ultrasónico. Estos sistemas monitorizan el proceso protegiendo la integridad tanto de la herramienta como de la pieza.

La herramienta empleada para la operación de taladrado (Figura 3, b) tiene un diámetro de $6 \mathrm{~mm}$, el tamaño de grano es de $107 \mu \mathrm{m}$ con una concentración de diamante medio y una unión electrodepositada de grado medio-alto. Mientras que la herramienta de fresado (Figura 3, c) tiene un diámetro de $10 \mathrm{~mm}$, tamaño de grano de $107 \mu \mathrm{m}$ con una concentración de diamante medio y una unión metálica de grado medio. El material mecanizado en ambas tandas de ensayos es el Óxido de Aluminio (Alúmina) con una pureza del 99.9\%.

Los parámetros medidos han sido las fuerzas de corte, la rugosidad superficial, la redondez de los agujeros y el desgaste de la herramienta. La medición de las variables de salida durante los ensayos experimentales se ha realizado utilizando los siguientes equipos:

- Las fuerzas de corte se han capturado mediante un dinamómetro de la marca Kistler modelo 9257-BA.

- La rugosidad superficial se ha medido después de cada ensayo en las operaciones de fresado y tras el conjunto de todos en el taladrado, empleand o para ello un rugosímetro de la marca Mitutoyo modelo SJ201P. Las mediciones se hicieron en la dirección de avance, $X$ en las operaciones de fresado, y $\mathrm{Z}$ en las de taladrado.

- La redondez se midió empleando una máquina de medir por coordenadas de la marca Zeiss modelo UPMC 850 CARAT, evaluando 3 planos de cada agujero como se describirá después.

- El desgaste se ha determinado con un microscopio óptico y un perfilómetro confocal.

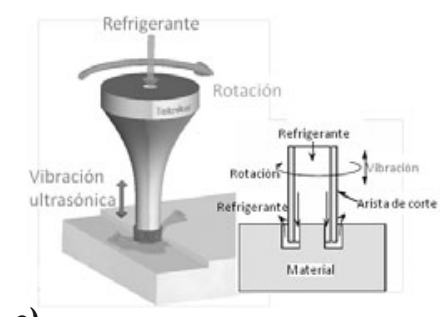

a)

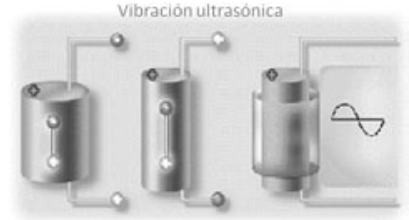

b)

Figura 1. Cerámicas piezoeléctricas.

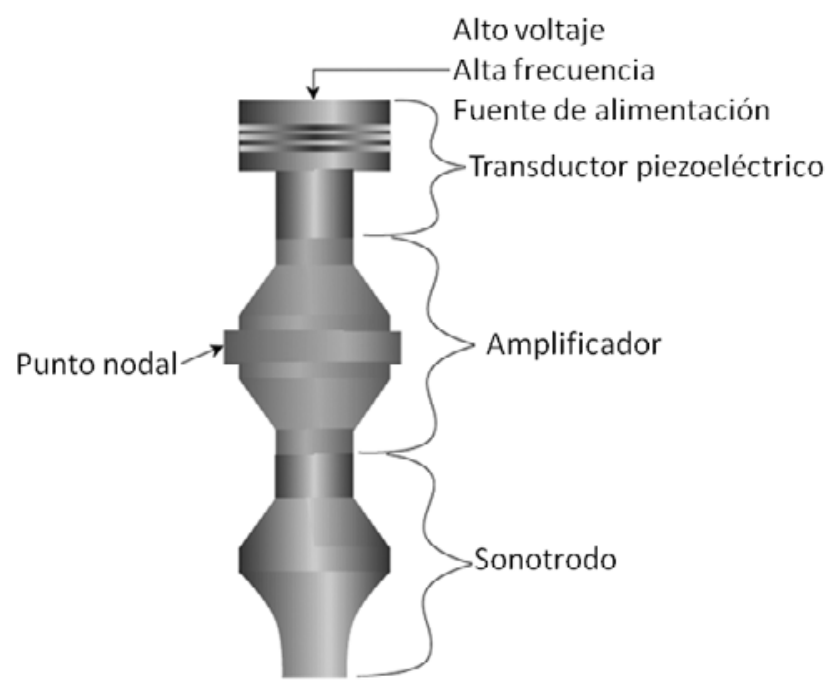

Figura 2. Imagen esquemática del sistema resonante del cabezal ultrasónico.

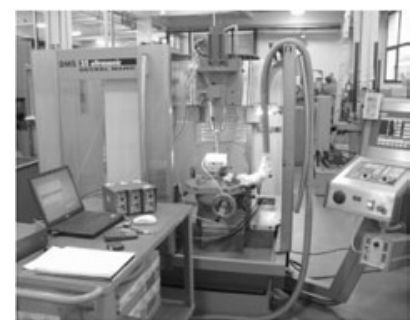

a)

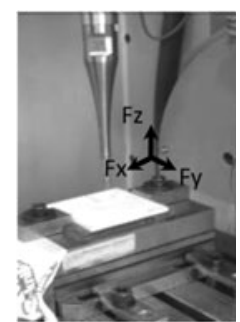

b)

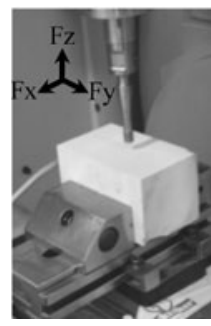

c)
Figura 3. Sistema experimental (a) para los ensayos de taladrado (b) $\mathrm{y}$ fresado (c).

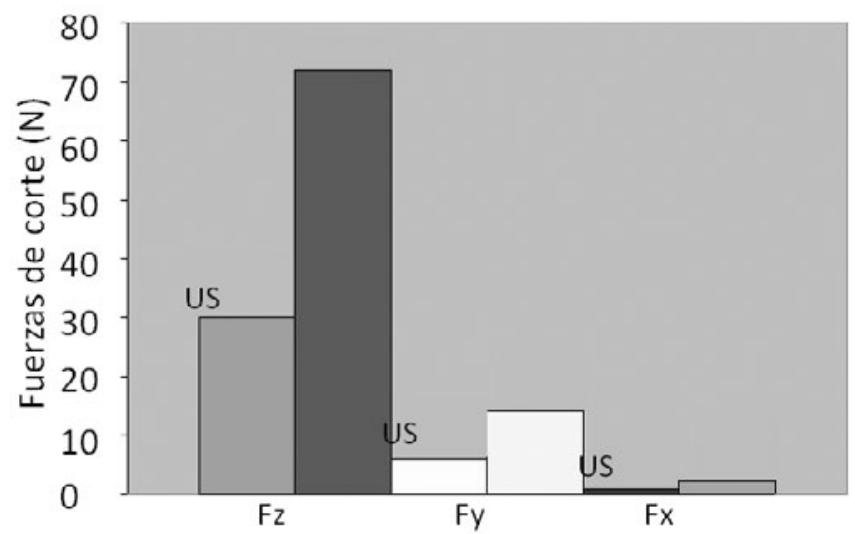

Figura 4. Promedio de fuerzas de corte con (US) y sin ultrasonidos durante el proceso de fresado. 
La medición de las fuerzas de corte se ha realizado montando la pieza mecanizada en el dinamómetro, realizando los ensayos con ese montaje, de forma que las fuerzas medidas con las soportadas por la pieza. Mientras que el resto de variables asociadas a la calidad (rugosidad, redondez, etc) se han realizado a posteriori en las muestras mecanizadas.

Tanto los ensayos de taladrado como los de fresado, en condiciones de acabado, se han realizado con y sin asistencia de los ultrasonidos, con el objeto de comparar el comportamiento del proceso de RUM frente a una operación similar al rectificado.

Las condiciones de vibración se han mantenido constantes y son las indicadas a continuación (ver tabla I):

TABLA I. PARÁMETROS DE VIBRACIÓN DE LOS ENSAYOS.

\begin{tabular}{|c|c|c|c|}
\hline Herramienta & $\begin{array}{c}\text { Frecuencia } \\
(\mathbf{H z})\end{array}$ & Potencia $(\boldsymbol{W})$ & Amplitud $(\boldsymbol{\mu m})$ \\
\hline Taladrado & 20600 & 10 & 30 \\
\hline Fresado & 20650 & 6 & 15 \\
\hline
\end{tabular}

Además, con objeto de observar el efecto de las condiciones de corte en estos dos procesos, fresado y taladrado, se ha creado un plan de ensayos mediante el método del diseño de experimentos (DOE) que posteriormente se analiza mediante el método de análisis de la varianza (ANOVA). Por una parte, en el proceso de taladrado se ha decidido realizar un factorial completo de dos niveles con tres variables con una sola repetición y 3 repeticiones en el punto central, resultando en 11 ensayos de taladrado. Mientras que en el caso del fresado, se ha llevado a cabo un factorial completo de dos niveles con cuatro variables con tres repeticiones y 3 repeticiones en el punto central, por lo que se han realizado 57 ensayos. Los detalles de las condiciones de corte se resumen en la tabla II.

De estos ensayos se analizan diferentes parámetros. Por ejemplo, en el caso del fresado se han analizado la fuerza de corte de avance $(\mathrm{Fx})$, radial $(\mathrm{Fy})$, axial $(\mathrm{Fz})$, la rugosidad $(\mathrm{Ra})$ y el desgaste. Por otro lado, durante los ensayos de taladrado, los parámetros medidos han sido la fuerza de corte axial (Fz), la rugosidad $(\mathrm{Ra})$ y la redondez.

Todos estos resultados se han analizado empleando el método de análisis de varianza (ANOVA) con el software STATGRAPHICS (42), mostrándose las correlaciones entre las variables de entrada y salida. En el caso del desgaste de la herramienta, el análisis se ha realizado por inspección visual y medida de los granos de la herramienta en varias zonas de la herramienta. En los capítulos siguientes se detallan los resultados y las conclusiones obtenidas con el conjunto de los ensayos realizados.

\section{RESULTADOS EXPERIMENTALES}

\subsection{Resultados de los ensayos de fresado}

A continuación se muestran los resultados de los ensayos experimentales realizados para el caso de fresado. En primer lugar, se va establecer la influencia de los parámetros de corte en la reducción de los esfuerzos de corte y la rugosidad inherentes al proceso de RUM en operaciones de planeado en fresado. Posteriormente, se analizará el comportamiento de las variables de salida en función de los cambios en las variables de entrada y por último se analizará el desgaste.

\subsubsection{ANÁLISIS DE LAS FUERZAS DE CORTE}

Los resultados que se muestran en la figura 4, promedios de los valores de las fuerzas, indican una clara reducción de las fuerzas de corte en el proceso con vibración frente al proceso sin vibración ultrasónica.

Estas reducciones suponen entre un 15-77 \%, 30-160\% y $20-97 \%$ de reducción en las fuerzas de corte de avance $(\mathrm{Fx})$, radial $(\mathrm{Fy})$ y axial $(\mathrm{Fz})$ respectivamente. Estas reducciones de las fuerzas de corte ocurren de acuerdo con los parámetros de corte de la forma que se indica a continuación (ver figura 5).

\begin{tabular}{|c|c|c|c|c|c|}
\hline & & \multicolumn{4}{|c|}{ VARIABLES DE ENTRADA } \\
\hline & Niveles & $\begin{array}{l}\text { Velocidad de giro (A) } \\
\text { (rpm) }\end{array}$ & $\begin{array}{c}\text { Avance } \\
\text { (B) } \\
\text { (mm/min) }\end{array}$ & $\begin{array}{l}\text { Profundidad de corte axial } \\
\text { (C) }(\mathrm{mm})\end{array}$ & $\begin{array}{l}\text { Profundidad de corte } \\
\text { radial (D) (mm) }\end{array}$ \\
\hline \multirow{4}{*}{ 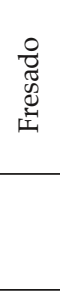 } & -1 & $2500 \mathrm{rpm}$ & 100 & 0.050 & 2.500 \\
\hline & 0 & $3250 \mathrm{rpm}$ & 200 & 0.100 & 3.750 \\
\hline & +1 & $4000 \mathrm{rpm}$ & 300 & 0.150 & 5 \\
\hline & & $\begin{array}{c}\text { Velocidad de giro (A) } \\
\text { (rpm) }\end{array}$ & $\begin{array}{l}\text { Avance (B) } \\
(\mathrm{mm} / \mathrm{min}) \\
\end{array}$ & Paso de taladrado $(\mathrm{C})(\mathrm{mm})$ & \\
\hline \multirow{3}{*}{$\begin{array}{l}\frac{8}{\pi} \\
\frac{\pi}{\pi} \\
\frac{\pi}{\pi} \\
\frac{\pi}{\pi}\end{array}$} & -1 & $3500 \mathrm{rpm}$ & 4 & 0.04 & \\
\hline & 0 & $4250 \mathrm{rpm}$ & 6.5 & 0.07 & \\
\hline & +1 & $5000 \mathrm{rpm}$ & 9 & 0.1 & \\
\hline
\end{tabular}


En cada gráfica se muestra la reducción de cada componente de fuerza en porcentaje respecto al proceso de mecanizado sin ultrasonidos en función de los cambios en los parámetros de corte que figuran en el eje horizontal. Los parámetros de corte varían entre su valor alto y bajo, produciendo las correspondientes variaciones en las fuerzas. La mayor pendiente en la recta de comportamiento de cada factor o parámetro de corte indica una mayor influencia de este factor en el valor de la salida correspondiente.

Las gráficas de la figura 5 muestran cómo afectan de distinta manera los parámetros de corte a la reducción de las componentes de fuerza. La componente $\mathrm{Fz}$ es la de mayor valor absoluto, siendo la más importante del proceso de corte, por ese motivo el estudio de su reducción es especialmente importante. En este sentido, la reducción es mayor cuando la velocidad de corte es mayor, pero decrece a medida que aumenta el avance y las profundidades axial y radial de corte, confirmándose la aplicabilidad del RUM a operaciones de fresado de planeado en condiciones de acabado.

La reducción de las fuerzas de corte se produce debido a la acción de la vibración ultrasónica, que es uno de los principales beneficios de esta tecnología.

\subsubsection{ANÁLISIS DE LA RUGOSIDAD SUPERFICIAL}

La rugosidad se midió después de cada ensayo y se compararon los resultados obtenidos con los obtenidos en las mismas condiciones sin ultrasonidos. La figura 6 muestra el promedio de los valores de la rugosidad de forma comparativa, para dar una idea del orden de magnitud de la mejora obtenida. La evolución de la rugosidad confirma lo apuntado en estudios previos (43), observándose que con el proceso RUM se obtiene una reducción de la misma, llegando en este caso hasta el $21 \%$.

El factor más importante en esta reducción es la propia vibración ultrasónica, y en cuanto a la dependencia de los parámetros de corte, subrayar que la reducción es mayor con el incremento de $a_{p}$ y $a_{e^{\prime}}$ tal y como se muestra en la siguiente figura 7.

La figura 7 refleja la importancia de 3 factores principalmente: la profundidad de corte axial $\left(a_{p}\right)$ y radial $\left(a_{e}\right)$, y el avance. A medida que el $a_{p}$ aumenta, la reducción de la rugosidad lo hace también; con profundidades de pasada muy pequeñas se ha observado poca reducción, y esto es debido a que la profundidad de pasada es muy similar a la amplitud de vibración, causando marcas en la superficie de la pieza. Por este motivo, se puede concluir que el $\mathrm{a}_{\mathrm{p}}$ no puede tomar valores del orden de magnitud de la amplitud de vibración si se quieren obtener buenos acabados superficiales. A su vez, la amplitud de vibración es regulable en función de la frecuencia de vibración de la herramienta por lo que se puede ajustar la amplitud y el $a_{p}$ para obtener los resultados más adecuados en cada caso.

\subsubsection{MODELOS OBTENIDOS DE LOS EXPERIMENTOS}

En relación con el comportamiento de las variables de salida, $F_{x^{\prime}} F_{y^{\prime}} F_{Z}$ y $R_{a^{\prime}}$ estos son los resultados para el rango de valores de las variables de entrada ensayadas que corresponden a condiciones de acabado:
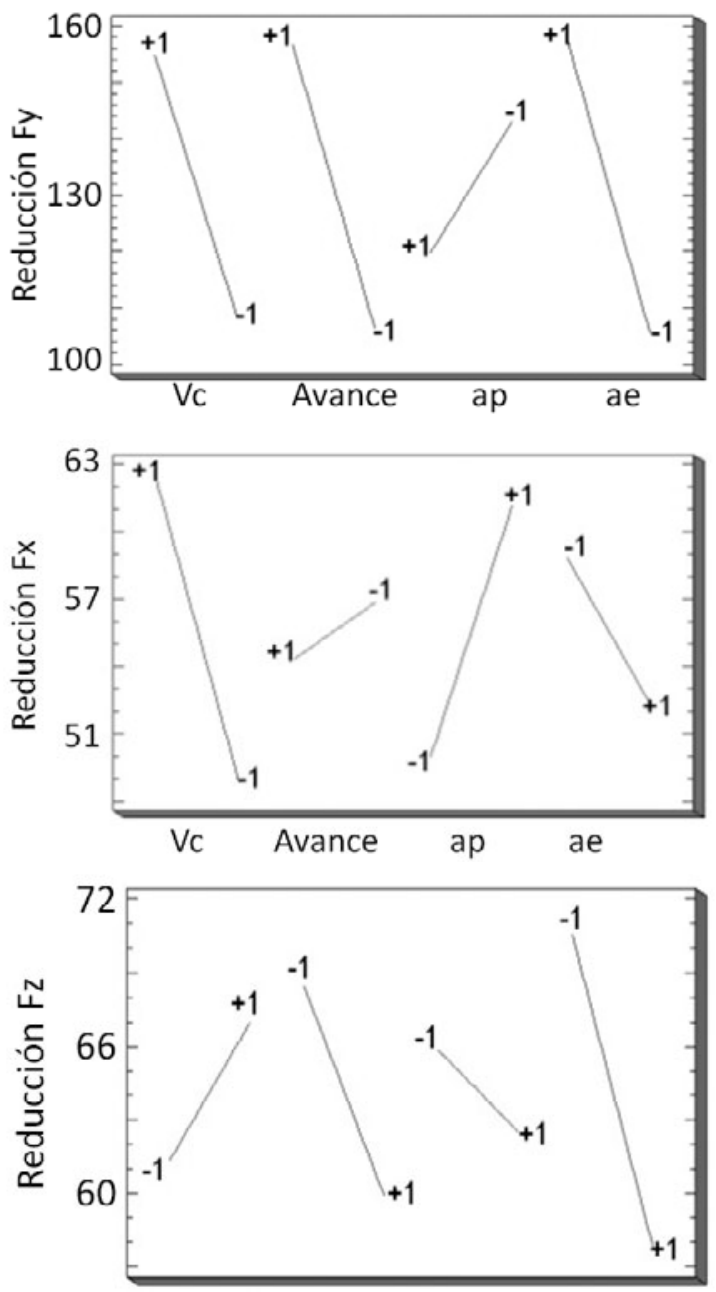

Figura 5. Efectos de los parámetros de corte en la reducción (\%) de los esfuerzos de corte.

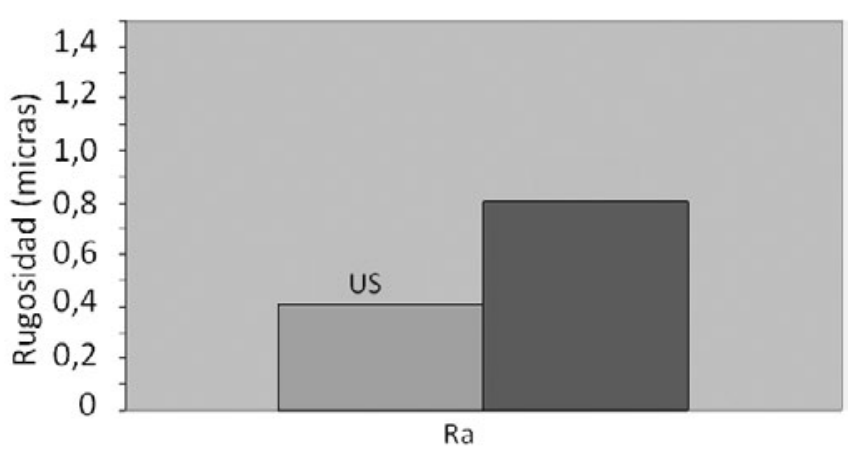

Figura 6. Promedio de rugosidad con (US) y sin ultrasonidos.
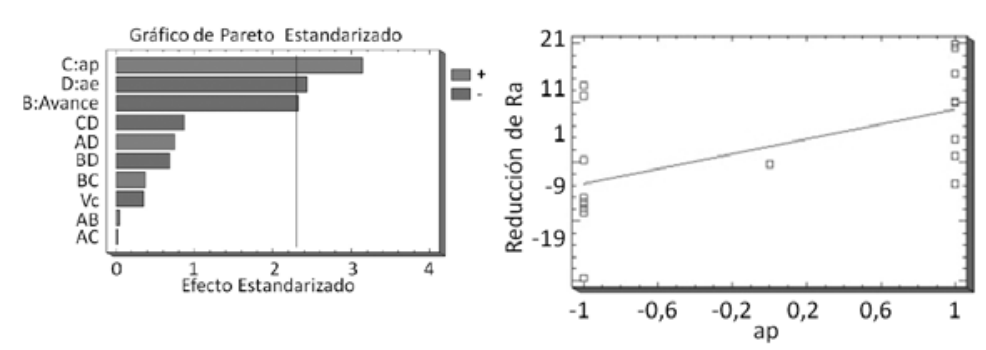

Figura 7. Efectos de los parámetros de corte en la reducción (\%) de Ra. 


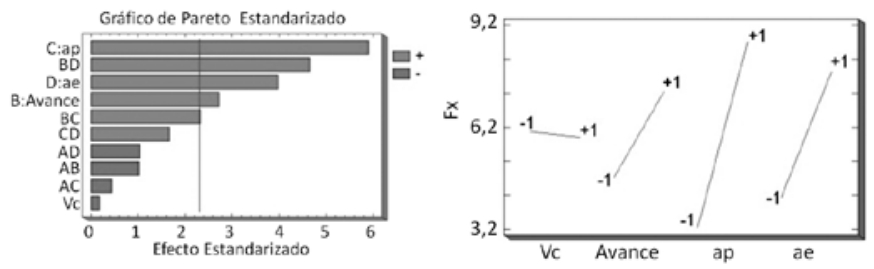

Figura 8. Efectos de los parámetros de corte en la fuerza de corte Fx.
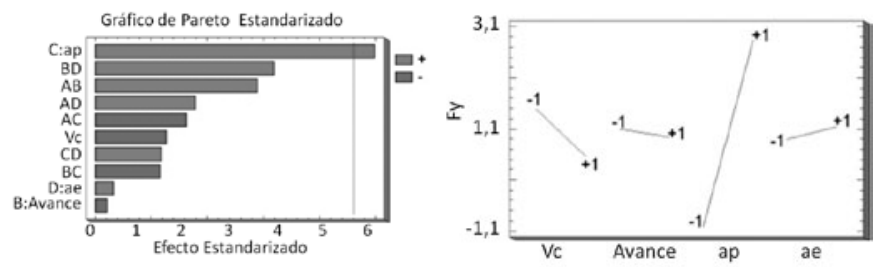

Figura 9. Efectos de los parámetros de corte en la fuerza de corte Fy.

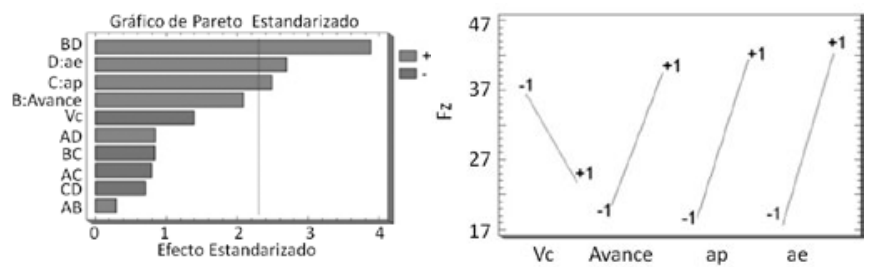

Figura 10. Efectos de los parámetros de corte en la fuerza de corte Fz.
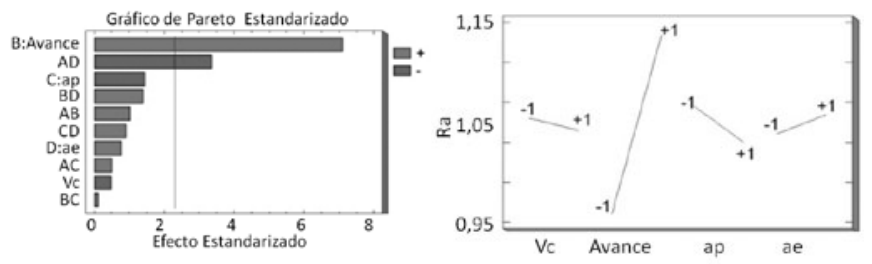

Figura 11. Efectos de los parámetros de corte en la rugosidad superficial.

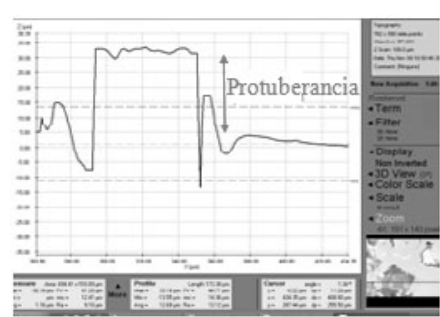

a)

Figura 12. Ejemplo de una medición 3D de las protuberancias de los granos de diamante en la superficie de corte de la herramienta mediante un perfilómetro confocal: a) perfil de una protuberancia y b) mapa 3D de la superficie de corte.

\subsubsection{Fuerza de avance: $F x$}

Las correlaciones de la componente $\mathrm{F}_{\mathrm{x}}$ se establecen tal $\mathrm{y}$ como aparece en las gráficas que se muestran en la figura 8.

Los resultados obtenidos muestran que los factores más importantes son $\mathrm{a}_{\mathrm{p}}(\mathrm{C}), \mathrm{BD}$ (producto avance $\mathrm{y} \mathrm{a}_{\mathrm{e}}$ ), $\mathrm{a}_{\mathrm{e}}(\mathrm{D})$ y avance (B). Así, la $F_{x}$ decrece con el aumento del avance, $a_{p}$ y $a_{e^{\prime}}$ siendo la influencia de la velocidad de corte (A) prácticamente inapreciable.

\subsubsection{Fuerza radial: Fy}

Por su parte, el análisis de los efectos de los parámetros de corte y el modelo obtenido para la componente $\mathrm{F}_{\mathrm{Y}}$, presenta los siguientes resultados reflejados en la figura 9.

En este caso, el factor más importante es el $\mathrm{a}_{\mathrm{p}^{\prime}} \mathrm{y}$ a medida que este aumenta lo hace también la componente $F_{Y}$ de la fuerza de corte. El resto de factores tienen una influencia despreciable en esta componente de fuerza.

\subsubsection{Fuerza axial: $F z$}

Esta es la componente de la fuerza de mayor valor absoluto y mayor importancia en el proceso. Los resultados referentes a la misma se resumen a continuación (ver figura 10).

Los factores más relevantes que contribuyen al comportamiento de la $\mathrm{F}_{\mathrm{Z}}$ son las variables de carga de viruta $\left(\mathrm{a}_{\mathrm{p}} \mathrm{y} \mathrm{a}_{\mathrm{e}}\right)$, aparte del avance introducido por el término BD, el cual se obtiene de la multiplicación del avance y el $\mathrm{a}_{\mathrm{e}}$. La $\mathrm{F}_{\mathrm{z}}$ aumenta a la vez que lo hace el avance y las profundidades de corte axial y radial. La velocidad de corte tiene una influencia menor en este caso.

\subsubsection{Rugosidad superficial $\mathrm{Ra}$}

Esta variable tiene una gran importancia en los procesos de fabricación ya que da la calidad superficial final de las piezas. La rugosidad se ha medido en la dirección del avance (eje X) y los ensayos han arrojado los resultados que se puede apreciar en la figura 11, el factor más importante en el comportamiento de la rugosidad es el avance, empeorando la rugosidad a medida que el avance se incrementa. El resto de variables de entrada estudiadas apenas tienen influencia en la rugosidad superficial obtenida tras el mecanizado.

\subsubsection{DESGASTE}

Otro parámetro importante a medir en este análisis ha sido el desgaste de la herramienta que define la vida útil de este. Para ello se han realizado medidas 3D de las protuberancias de los granos de diamante en la superficie de corte de la herramienta después de cada ensayo (ver figura 12) empleando un perfilómetro confocal. Cuantas mayores protuberancias haya se entiende que los granos de diamante adheridos en la superficie de corte de la herramienta han sufrido menos desgaste por lo que se consiguen mayores tasas de arranque de material.

La Figura 13 muestra la comparativa de protuberancias medidas en dos ensayos después de un proceso de rectificado y un proceso RUM de la Alumina. Los resultados muestran que las dimensiones iniciales de los granos de diamante de unas 58 micras son reducidos entre un $41.3 \%$ y $48.2 \%$ en el caso del rectificado y entre un $26.7 \%$ y $34 \%$ en el caso del RUM. 
Estos resultados muestran que con el proceso RUM se obtienen menores desgastes de herramienta por lo que hay más granos de diamante para obtener tasas de arranque de material mayores.

\subsection{Resultados de los ensayos de taladrado}

A continuación se muestran los resultados de los ensayos experimentales realizados para el proceso de taladrado. En primer lugar, se va a analizar la influencia de los parámetros de corte en la reducción de los esfuerzos de corte y la rugosidad inherentes al proceso de RUM en operaciones de taladrado. Posteriormente, se analizará el comportamiento de las variables de salida en función de los cambios en las variables de entrada, analizándose también la redondez.

\subsubsection{ANÁLISIS DE LAS FUERZAS DE CORTE}

Los resultados de los experimentos realizados indican una clara reducción de fuerzas de corte (ver figura 14) en los casos de utilización de mecanizado con ultrasonidos frente al taladrado sin ultrasonidos, al igual que se ha observado en el proceso de fresado.

Este beneficio se obtiene mediante un proceso similar al "peck drilling" que se basa en un taladrado paso a paso donde la broca se levanta cada cierto tiempo para evacuar la viruta y limpiar el agujero. El factor más importante que causa esta reducción es el paso de taladrado, tal y como aparece en la siguiente figura 15.

La fuerza de corte $\mathrm{F}_{\mathrm{z}}$ es la más influyente en una operación de taladrado y por ello su reducción es especialmente importante. La figura 15 muestra cómo los parámetros de corte afectan a la modificación de esta componente de fuerza. Así, se puede concluir que la reducción es mayor cuanto mayor sea el paso de taladrado, el avance y la velocidad de corte.

$\mathrm{El}$ hecho de que mayores pasos de taladrado resultan en reducciones más importantes de los esfuerzos, sugiere una mejora de la productividad que se obtendría a medida que se utilicen mayores pasos de taladrado en el proceso RUM con la consiguiente reducción de esfuerzos axiales.

\subsubsection{ANÁLISIS DE LA RUGOSIDAD SUPERFICIAL}

La rugosidad se ha medido después de cada ensayo y se han comparado los taladros realizados con y sin ultrasonidos. La evolución de la rugosidad confirma lo apuntado en estudios previos (42), observándose una clara reducción de la misma (ver figura 16) en el proceso de RUM, llegando en algunos casos hasta el $80 \%$.

La dependencia de los parámetros de corte en la reducción de la rugosidad no se ha obtenido de manera explícita tras el análisis del diseño de experimentos, concluyéndose que la principal variable de influencia en el valor final de la rugosidad es la propia vibración ultrasónica y los parámetros asociados a la misma, como la amplitud, la frecuencia, etc.

\subsubsection{ANÁLISIS DE LA REDONDEZ}

A la hora de cuantificar la calidad de los taladrados realizados (Figura 17 a) se han realizado medidas de redondez o circularidad de los agujeros realizados sin y con ultrasonidos. Las mediciones se han llevado a cabo en una máquina de

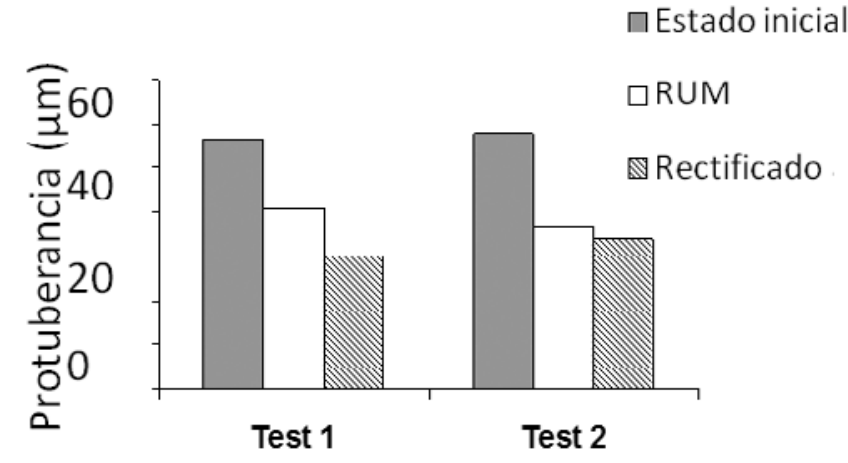

Figura 13. Protuberancias de los granos de diamante después de 18 metros de mecanizado.

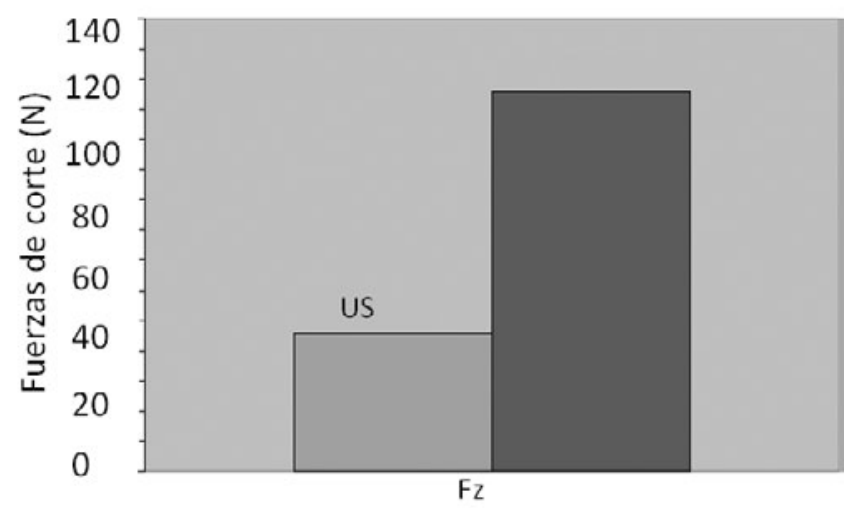

Figura 14. Promedio de fuerza de avance $(\mathrm{Fz})$ con y sin ultrasonidos en taladrado.

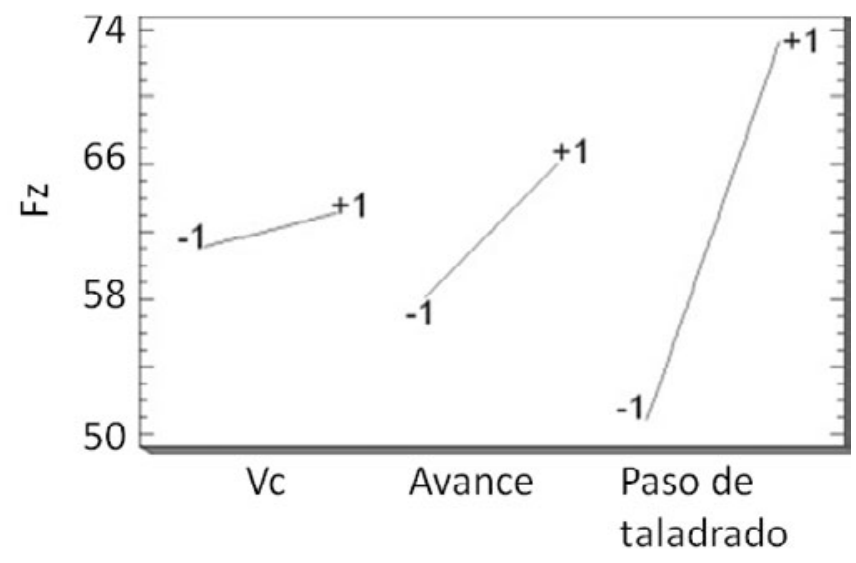

Figura 15. Efectos de los parámetros de corte en la reducción $(\%)$ de las fuerzas en Fz para el taladrado.

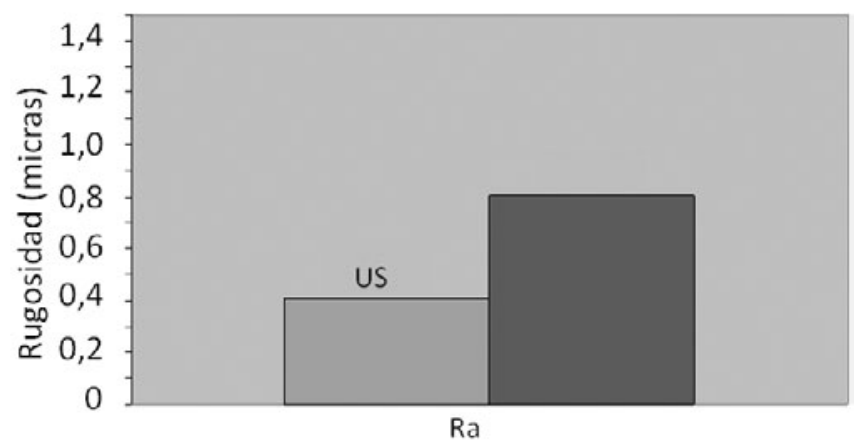

Figura 16. Promedio de Ra con (US) y sin ultrasonidos. 


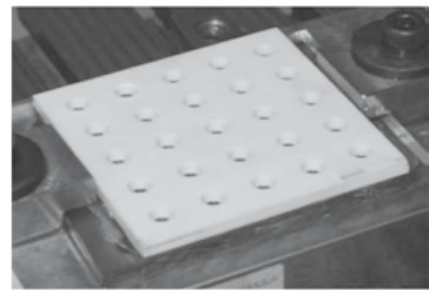

a)

Figura 17. a) Probeta obtenida tras los ensayos y b) imagen de una de las medidas de redondez.
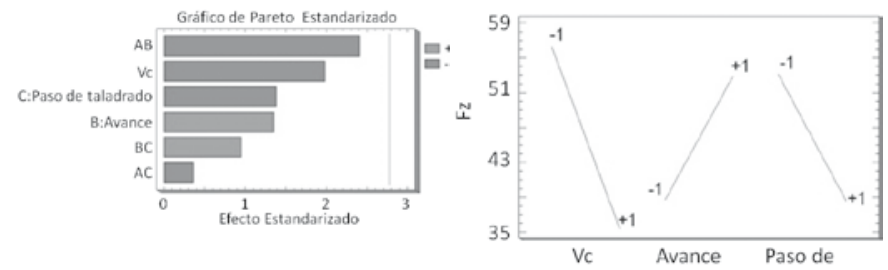

Figura 18. Efectos de los parámetros de corte en la fuerza de avance (Fz) en el taladrado.
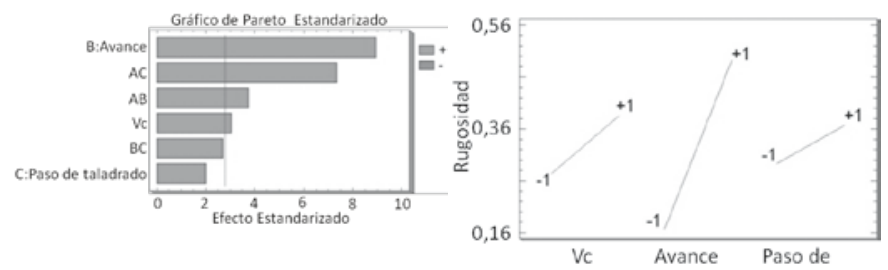

Figura 19. Efectos de los parámetros de corte en la rugosidad ( $\mathrm{Ra}$ ) en el taladrado.

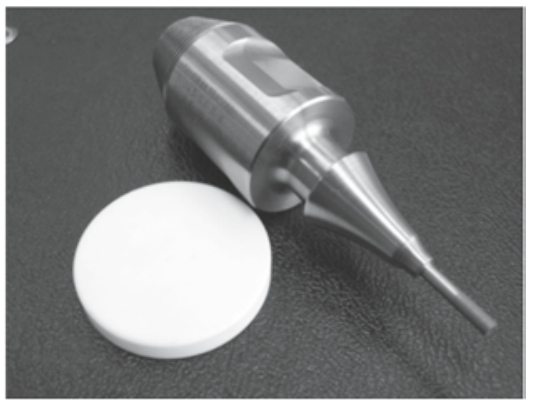

Figura 20. Material de partida (Alúmina) y herramienta de diamante electrodepositado de $Ø 3 \mathrm{~mm}$ con tamaño de grano D76. medir por coordenadas tomando 590 puntos a lo largo de la circunferencia (Figura 17 b) y midiendo tres planos en tres alturas diferentes en cada agujero: a la entrada, en un plano medio y a la salida.

Los resultados muestran una mejora en la redondez de los agujeros con ultrasonidos respecto a los realizados sin ultrasonidos. La siguiente tabla III resume los resultados obtenidos en las mediciones.

Como se puede comprobar, exceptuando la entrada del ensayo 2 y el plano central del ensayo 6, todos los planos tienen una mejor redondez con ultrasonidos. Este hecho indica la capacidad del RUM para la mejora de la precisión en el taladrado en condiciones de acabado.

\subsubsection{MODELOS OBTENIDOS DE LOS EXPERIMENTOS}

Respecto al comportamiento de las variables de salida en los ensayos de taladrado, $\mathrm{F}_{\mathrm{z}}$ y $\mathrm{R}_{\mathrm{a}^{\prime}}$ se muestran a continuación los resultados referentes a su relación con el comportamiento de las variables de salida en el rango de valores de las variables de entrada ensayadas, que corresponden a condiciones de acabado.

\subsubsection{Fuerza axial: Fz}

Los resultados del comportamiento de $\mathrm{F}_{\mathrm{Z}}$ en función de los parámetros de entrada se muestran en la siguiente figura 18.

Los factores que contribuyen de manera más importante al comportamiento de $\mathrm{F}_{\mathrm{z}}$ son la velocidad de corte $\mathrm{y}$ el avance introducidos por el factor $\mathrm{AB}$. La fuerza $\mathrm{F}_{\mathrm{z}}$ aumenta a medida que lo hace el avance y decrece con la velocidad de corte y el paso de taladrado.

\subsubsection{Rugosidad superficial $\mathrm{Ra}$}

Esta variable ha sido medida en la dirección de avance o axial del agujero, eje $Z$, presentando los siguientes resultados que se observan en la figura 19.

En vista del análisis de los resultados, se puede concluir que el factor más importante en el comportamiento de la rugosidad es el avance, y a medida que el avance crece se produce un empeoramiento de la rugosidad obtenida. El resto de variables de entrada no tienen una influencia significativa en la rugosidad final obtenida.

\subsection{Tamaño de las virutas}

En paralelo con la experimentación, se efectuaron varias medidas del tamaño de las virutas obtenidas durante el mecanizado. Este análisis se ha realizado con muestras de

TABLA III. RESULTADOS DE REDONDEZ DE LOS 3 ENSAYOS SELECCIONADOS.

\begin{tabular}{|c|c|c|c|c|c|c|}
\hline & \multicolumn{6}{|c|}{ Redondez (mm) } \\
\hline & \multicolumn{2}{|c|}{ Ensayo 1} & \multicolumn{2}{|c|}{ Ensayo 2} & \multicolumn{2}{|c|}{ Ensayo 3} \\
\hline & Con US & Sin US & Con US & Sin US & Con US & Sin US \\
\hline Entrada & 0.006 & 0.003 & 0.002 & 0.002 & 0.001 & 0.005 \\
\hline Mitad & 0.002 & 0.004 & 0.005 & 0.003 & 0.003 & 0.005 \\
\hline Salida & 0.004 & 0.005 & 0.003 & 0.004 & 0.002 & 0.005 \\
\hline
\end{tabular}


taladrina que fueron analizadas, comparando el tamaño de las partículas obtenidas en los ensayos con y sin ultrasonidos. El proceso se ha llevado a cabo mediante la técnica conocida como "filtrografía" y a continuación se muestra un ejemplo con una de las mediciones:

Estos análisis incluyen información acerca de las partículas metálicas provenientes del desgaste de la herramienta, fibras de restos del filtro de taladrina de la máquina de RUM, y partículas no metálicas que corresponden a las virutas cerámicas del material mecanizado, es decir, de Óxido de Aluminio. Estos ensayos confirman una reducción del tamaño de viruta de hasta el $57 \%$.

\section{APLICACIÓN INDUSTRIAL}

Con objeto de verificar las ventajas y beneficios de la tecnología del Mecanizado por Ultrasonidos Rotatorio se ha mecanizado una pieza real del mismo material que el empleado en los ensayos experimentales, la Alúmina. La pieza en cuestión está diseñada por Sauer Ultrasonic (DMG) y forma parte de un anillo de ajuste.

El mecanizado de dicha pieza incluye las operaciones estudiadas en el presente estudio, es decir, operaciones de taladrado y de fresado. El mecanizado se ha llevado a cabo con una sola herramienta de diamante electrodepositado de Ø3mm con tamaño de grano D76.

Los parámetros de mecanizado utilizados en las diferentes operaciones son los indicados en las tablas IV y V.

En lo referente a las condiciones de vibración de la herramienta, la frecuencia empleada ha sido $20650 \mathrm{~Hz}$ y una potencia de $6 \mathrm{~W}$, obteniéndose una amplitud de vibración de $10 \mu \mathrm{m}$ aproximadamente.

Después de haber invertido 1h 10min de mecanizado, el resultado final ha sido satisfactorio y cumple los requisitos en cuanto a tolerancias dimensionales y rugosidad superficial. En cuanto a las dimensiones finales de la pieza, se verificó un error máximo de $20 \mu \mathrm{m}$ debido principalmente al desgaste de la herramienta durante el proceso. En lo referente a la rugosidad media se obtuvo un valor inferior a $1 \mu \mathrm{m}$ Ra. Por otro lado, no se observaron grietas ni daños superficiales.

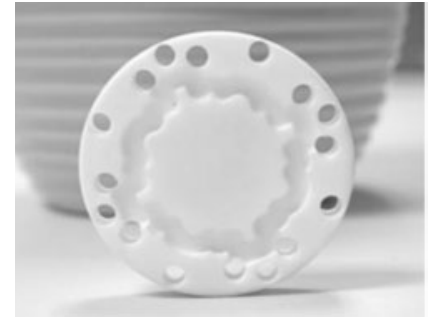

a)

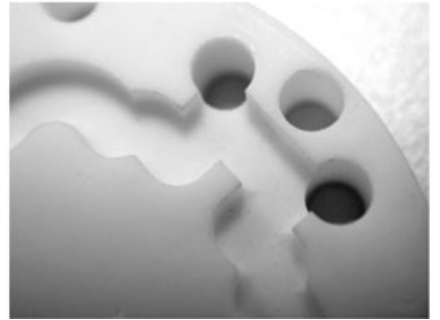

b)
Figura 21. Resultado final de la pieza test: a) pieza completa a la izquierda y b) detalle en la parte derecha.

Aunque en este trabajo se ha mostrado un único caso industrial, el mecanizado de un elemento de un anillo de ajusto de Alúmina, los resultados obtenidos se pueden trasladar a otras piezas industriales fabricadas en materiales duros y frágiles como cerámicas técnicas, vidrios, piedra, Silicio, piedras preciosas, etc. que se han podido analizar con la máquina RUM en Tekniker. En un futuro cercano se espera que, con los beneficios expuestos, esta tecnología pueda facilitar el empleo de distintas cerámicas técnicas y adentrarse en otros sectores:

- Industria de los semiconductores: plaquitas (Wafer), elementos de refrigeración...en materiales como Silicio, Cuarzo Hialino, etc.

- Industria del automóvil: discos de freno, toberas de inyección, insertos de moldes de inyección...; en materiales como Nitruro de Silicio, Alúmina, metal duro, acero templado $(55 \mathrm{HRc})$, etc.

- Industria óptica: lentes cóncavas y convexas, espejos...; en materiales como Zafiro, Silicio, Zerodur y vidrios varios.

- Industria de la piedra: mármol, granito, Silestone, etc.

- Industria médica: articulaciones, coronas dentales...; en materiales cerámicos varios como Zirconia, Alúmina, etc.

- Varios: guías antidesgaste, rodamientos, pirometría, boquillas de soldadura, aisladores térmicos...; compuestos por materiales cerámicos.

TABla IV. CONDICIONES DE TAMAÑOS DE PARTíCULAS CON Y SIN ULTRASONIDOS.

\begin{tabular}{|c|c|c|c|c|}
\hline Partículas & Bajo & Medio & Alto & Tamaño medio $(\mu$ m) \\
\hline & Con Us/Sin Us & Con Us/Sin Us & Con Us/Sin Us & Con Us/Sin Us \\
\hline Metálico & Ok/Ok & & & $150 / 140$ \\
\hline No metálico & Ok/Ok & & & $40 / 70$ \\
\hline Fibras & Ok/Ok & & & $500 / 600$ \\
\hline
\end{tabular}

TABLA V. CONDICIONES DE CORTE DEL RUM PARA LA APLICACIÓN INDUSTRIAL.

\begin{tabular}{|c|c|c|c|c|}
\hline Proceso & $\begin{array}{c}\text { Velocidad de giro } \\
(\mathbf{r p m})\end{array}$ & $\begin{array}{c}\text { Avance } \\
(\mathbf{m m} / \mathbf{m i n})\end{array}$ & $\begin{array}{c}\text { Profundidad de corte } \\
\text { axial (mm) }\end{array}$ & $\begin{array}{c}\text { Profundidad de corte } \\
\text { radial (mm) }\end{array}$ \\
\hline Fresado & 4000 & 500 & 0.025 & 3 \\
\hline Taladrado & 4000 & 9 & 0.1 & 3 \\
\hline
\end{tabular}




\section{CONCLUSIONES}

Este trabajo presenta el comportamiento y los beneficios de la tecnología del Mecanizado por Ultrasonidos Rotatorio, verificando las ventajas del proceso en el mecanizado de alúmina en operaciones de fresado y taladrado. Los resultados confirman que el RUM es una tecnología interesante como alternativa al rectificado en el mecanizado de materiales duros y frágiles. Entre las principales conclusiones destacan las siguientes:

- Se produce una clara reducción de las fuerzas de corte debido a la vibración ultrasónica para las operaciones de fresado y de taladrado. Esta reducción de esfuerzos de corte previene defectos durante el mecanizado de los materiales duros y frágiles en general. Los parámetros de corte afectan de diferente manera a cada componente de la fuerza de corte, así, la reducción del esfuerzo axial es mayor con condiciones de mecanizado suaves (bajos $\mathrm{a}_{\mathrm{p}^{\prime}} \mathrm{a}_{\mathrm{e}} \mathrm{y}$ avance) en fresado y con largos pasos de taladrado en esta operación.

- La reducción de los esfuerzos de corte y el tamaño de las virutas, son las principales ventajas introducidas por el efecto de la vibración. El proceso con ultrasonidos produce virutas más pequeñas que sin ultrasonidos.

- Se observa así mismo, una mejora de la rugosidad de hasta el $20 \%$ en operaciones de fresado y del $80 \%$ en las de taladrado.

- El desgaste observado en los ensayos con ultrasonidos es considerablemente menor que en un proceso sin ultrasonidos por lo que hay más granos de diamante para obtener mayores tasas de arranque de material (MRR).

- Los resultados que arrojan las medidas de redondez son positivos; el proceso con ultrasonidos produce una mejor calidad en los taladros, avalando la aplicabilidad del RUM a operaciones de acabado en taladrado.

- Finalmente, se mecanizó una pieza test en el mismo material (Óxido de Aluminio) y se realizaron las mismas operaciones (fresado y taladrado) que en el estudio experimental. Los resultados confirman que el RUM es una tecnología interesante como alternativa al rectificado en el mecanizado de materiales duros y frágiles en condiciones de acabado.

- En definitiva, el RUM es un proceso prometedor para las operaciones de mecanizado de cerámicas estructurales de formas complejas en condiciones de acabado. Sus características pueden fomentar el uso de las cerámicas en un mayor número de aplicaciones industriales, debido a las posibilidades de aumentar las capacidades tanto de desarrollo como de diseño de productos de mayor valor añadido.

\section{AGRADECIMIENTOS}

Los autores quieren agradecer al Departamento de Industria del Gobierno Vasco-Eusko Jaurlaritza la ayuda recibida para la realización de este trabajo en el proyecto CIC MARGUNE del programa ETORTEK.

\section{BIBLIOGRAFÍA}

(1) Serratosa, J. M., La investigación en Ciencia de Materiales en España. Evolución en los últimos 30 años, Bol. Soc. Esp. Ceram. V., 49, 2, 129-138 (2010)

(2) Hu, P., Zhang, J.M., Pei, Z.J., Treadwell, C., Modeling of Material Removal Rate in Rotary Ultrasonic Machining: Designed Experiments, Journal of Materials Processing Technology 129, 339-344 (2002).

(3) Tsutsumi, C., Okano, K., Suto, T., High quality machining of ceramics, Journal of Materials Processing Technology 37, 639-654 (1993).

(4) Jahanmir, S., Ives, L.K., Ruff, A.W., Peterson, M.B., Ceramic Machining: Assessment of Current Pratice and Research Needs in the United States, NIST Special Publication 834 (1992).

(5) Kreuels, N., A view on the European Refractory Industry, Bol. Soc. Esp. Ceram. V. 48, 5, 245-254 (2009).

(6) Shou P., Present status and internationalization prospect of china float glass technology, Bol. Soc. Esp. Ceram. V. 47, 2, 117-121 (2008)

(7) Barrie, C., Why ceramics are a hot property, The Engineer, pp. 46-50, November 1986.

(8) R. Kamo, Ceramics play integral role in adiabatic's future, Ceramic Industry, pp. 26-29, July 1984 .

(9) J.W. MacBeth, Ceramic engine components reduce wear, friction, in Ceramic Industry, pp. 33-45, July 1984

(10) Anonymous, Ceramics shape up for heat engine applications, in: Advanced Materials \&Processes, pp. 21-22 (1995).

(11) Legge, P., Ultrasonic drilling of ceramics, Industrial Diamond Review 24 (278) 20-24 (1964).

(12) Anonymous, An improved ultrasonic machine tool for glass and ceramics, Industrial Diamond Review 26 (308).

(13) Machining without abrasive slurry, in: Ultrasonics, pp. 157-162, July 1966

(14) Pei, Z.J., Khanna, N., Ferreira, P.M., An investigation into rotary ultrasonic machining of structural ceramics- A review, Ceramic Engineering and Science Proceedings, 16 (1) 259278 (1995)

(15) Churi, N.J., Pei, Z.J., Shorter, D.C., Treadwell, C., Rotary Ultrasonic Machining of Silicon Carbide: Designed Experiments, International Journal of Manufacturing Technology and Management, 12 (1-3), 284 (2007)

(16) Treadwell, C., Pei, Z.J., 2003. Machining Ceramics with Rotary Ultrasonic Machining, Ceramic Industry, 39-42.

(17) Li, Z., Treadwell, C., Pei, Z.J., Drilling Small Holes in Hard-to-Machine Materials by Rotary Ultrasonic Machining, SME Technical Paper, TP04PUB137, 17p (2004).

(18) Churi, N.J., Pei, Z.J., Li, Z.C., Treadwell, C., Rotary Ultrasonic Machining of Titanium Alloy: A Feasibility Study, Proceedings of the ASME International Mechanical Engineering Congress and Exposition, Orlando, Florida, USA, IMECE2005-80254, 8p (2005).

(19) Cong W.L., Feng. Q, Pei, Z.J., Treadwell, C. Comparison of superabrasive tools in rotary ultrasonic machining of stainless steel. Proceedings of the ASME, v. 1, pp. 113-119 (2010).

20) Cong W.L., Feng. Q, Pei, Z.J., Treadwell, C. Drilling of silicon based solar panels: A review. Proceedings of the ASME, v. 1 pp. 309-316 (2010).

(21) Blake, P.N., Scattergood, R.O., Ductile-regime machining of germanium and silicon Journal of American Ceramic Society 73 949-957 (1990).

(22) Nakasuji, T., Kodera, S., Hara, S., Matsunaga, H., Ikawa, N., Shimada, S., Diamond turning of brittle materials for optical components, Annals of CIRP 39 (1) 89-92 (1990)

(23) Subramanian, K., Ramanath, S., Mechanism of material removal in the precisión grinding of ceramics, in: PED- vol. 58, Precision Machining: Technology and Machine Development and Improvement, ASME, pp. 1-19 (1992).

(24) Bifano, T.G., Dow, T.A., Scattergood, R.O., Ductile-regime grinding. A new technology for machining brittle materials, in: Transactions of the ASME, Journal of Engineering for Industry, vol. 113, pp. 184-189 (1991).

(25) Pei, Z.J., Ferreira, P.M., Modeling of ductile-mode material removal in rotary ultrasonic machining. International Journal of Machine Tools \& Manufacture 38 1399-1418 (1998).

(26) Kubota, M., Tamura, Y., Shimamura, N., Ultrasonic machining with a diamond impregnated tool. Bulletin of Japan Society of Precision Engineering II (3)(1977) 127-132.

27) Markov, A.I., Ustinov, I.D., A study of the ultrasonic diamond drilling of non-metallic materials, in: Industrial Diamond Review, March, pp. 97-99 (1972).

(28) Markov, A.I., Ultrasonic drilling and milling of hard non-metallic materials with diamond tools, Machine \& Tooling 48 (9) 45-47 (1977).

(29) Prabhakar, D., Ferreira, P.M., Haselkorn, M., An experimental investigation of material removal rates in rotary ultrasonic machining, in: Transaction of the North American Manufacturing Reserach Institution of SME, vol. XX, pp. 211-218 (1992).

(30) Wang, H.M.S., Lin, L.Y., Improvement of rotary ultrasonic deep hole drilling of glass ceramicsZedour, in: Seminar of the $7^{\text {th }}$ International Precision Engineering 52 (12) 144-147 (1986).

(31) Gon, H, Fan, F.Z., Hu, X.T., Kinematic view of tool life in rotary ultrasonic side milling of hard and brittle materials. International Journal of Machine tools \& Manufacture. Vol. 50-3 pp. 303-307.

(32) Sánchez-Herencia, A.J., Baudín, C., Ceramic laminates with tailored residual stresses, Bol. Soc. Esp. Ceram. V. 48, 6, 311-320 (2009).

(33) Li, Z.C., Liang, W.C., Pei, Z.J., Treadwell, C.,. Edge-Chipping Reduction in RUM of Ceramics: FEA and Experimental Verification, International Journal of Machine Tools $\mathcal{E}$ Manufacture 46, 1469-1477 (2006).

(34) Prabhakar, D., Pei, Z.J., Ferreira, P.M., Haselkorn, A theoretical model for predicting Prabhakar, D., Pei, Z.J., Ferreira, P.M., Haselkorn, A theoretical model for predicting
material removal rates in rotary ultrasonic machining of ceramics, Transactions of the N.A.M.R.A.C. 21, 167-172 (1993)

(35) Pei, Z.J., Ferreira, P.M., Haselkorn, Plastic flow in rotary ultrasonic machining of ceramics, Journal of Materials Processing Technology 48 771-777 (1995).

(36) Pei, Z.J., Ferreira, Modeling of ductile-mode material removal in rotary ultrasonic machining. International Journal of Machine Tools \& Manufacture 38 1399-1418 (1998).

(37) Pei, Z.J., Prabhakar, D., Ferreira, P.M., Haselkorn, A mechanistic approach to the prediction of material removal rates in rotary ultrasonic machining, Journal of Engineering for Industry 117 142-151 (1995).

(38) Petrukha, P.G. et al., Ultrasonic diamond drilling of deep holes in brittle materials, J. Russian Eng. 50 (10 70-74 (1970).

(39) Cleave, D.V., Ultrasonics gets bigger jobs in machining and welding, Iron Age 218 (II) 69-72 (1976).

(40) Graff, K.F., Ultrasonic machining, Ultrasonics 103-109 (1975).

(41) Vicario I, Gonzalo O, Bengoetxea I., "A feasibility study for rotary ultrasonic machining of aluminium oxide ceramics". $2^{\text {nd }}$ International meeting of Abrasion. Chalons en of aluminium oxide cera

(42) STATGRAPHICS software and documentation. http:/ / www.statgraphics.com.

(43) Wu, Y.B., Nomura, M., Feng, Z.J. and Kato, M.. “Modelling of Grinding Force in Constantdepth-of-cut Ultrasonically Assisted Grinding". Material Science Forum Vols. 471-472 pp.101-106 (2004).

Recibido: 10/11/2010

Aceptado: $13 / 06 / 2012$ 